\title{
IDENTIFICACIÓN DE UNA TÉCNICA DE ELABORACIÓN DE BOTELLAS MOCHE DE ASA ESTRIBO EN EL CASTILLO DE SANTA, COSTA NORTE DE PERÚ
}

\section{IDENTIFYING TECHNIQUES USED TO CONSTRUCT MOCHE STIRRUP SPOUT BOTTLES FROM EL CASTILLO DE SANTA, NORTHERN COAST OF PERU}

\section{Jorge Gamboa VelÁsquez*}

\begin{abstract}
El artículo describe una modalidad de elaboración de botellas de asa estribo identificada en El Castillo de Santa, un asentamiento de la segunda mitad del Período Intermedio Temprano (300-600 DC) ubicado en Ancash, costa norte de Perú. El análisis de la cerámica Moche III recuperada en ese sitio por el Proyecto Santa de la Universidad de Montreal permitió al autor identificar una técnica de manufactura de botellas distinta a la hasta ahora conocida para la sociedad Moche. Los resultados de este trabajo muestran la existencia de distintos métodos y cadenas operativas en la fabricación de las botellas de asa estribo, contribuyendo al reconocimiento de la diversidad regional y temporal existente al interior de las tecnologías de producción de la cerámica Moche.
\end{abstract}

Palabras clave: valle de Santa, Moche III, producción artesanal, botellas de asa estribo

This report describes a previously unknown technique for constructing Moche stirrup spout bottles discovered at El Castillo de Santa, a Moche settlement that flourished in the second half of the Early Intermediate Period (300-600 AD) on the northern coast of Peru. The site has been studied in recent years by the Santa Valley Project of the Université de Montreal. The author presents the specific steps involved in the technique and distinguishes it from other methods for making stirrup spouts known to date. This study demonstrates that multiple methods existed for producing Moche bottles and contributes to our understanding of diachronic and synchronic diversity in Moche ceramic technologies.

Key words: Santa Valley, Moche III Phase, craft production, stirrup spout vessels
Uno de los rasgos más sobresalientes en la producción prehispánica de cerámica en la costa norte de Perú fue la presencia constante de vasijas con golletes dispuestos sobre arcos tubulares. Las botellas de asa estribo no fueron exclusivas de la región, pero en ella su elaboración adquirió una notable continuidad, apareciendo desde el Horizonte Temprano (ca. 1000 AC) hasta el período de contacto con los europeos. Aun cuando esta forma cerámica tuvo una extraordinaria longevidad en el antiguo norte peruano, son las botellas de la tradición cultural Moche (200-750 DC) las que han captado el mayor interés de los investigadores por su calidad artística y carácter innovador en formas escultóricas y narrativas pictóricas.

Las sociedades Moche ocuparon la costa norte durante los primeros ocho siglos de nuestra era, basando parte de su economía en la producción artesanal especializada. Careciendo de sistemas de comunicación escrita fonética, los Moche produjeron algunas de las expresiones más descollantes del arte y la tecnología de América precolombina, erigiendo numerosos centros ceremoniales y urbanos de adobe y creando obras refinadas de metalurgia, textilería y pintura mural, así como elaborados registros pictóricos en línea fina (Uceda \& Mujica 1994, 2003; Pillsbury 2001; Jackson 2008; Quilter \& Castillo 2010; Trever et al. 2013). * Jorge Gamboa Velásquez, Proyecto Pañamarca - Área Monumental y Escuela de Arqueología de la Universidad Nacional Santiago Antúnez de
Mayolo, Huaraz, Perú, email: jgamboavelasquez@yahoo.com 
Las botellas de asa estribo Moche eran producidas por artesanos especializados. Aunque el empleo de moldes caracterizó al Período Moche, los alfareros continuaron desarrollando técnicas netamente manuales para elaborar algunas partes de estos objetos, conformados por la cámara y el asa estribo (compuesta a su vez por el arco tubular y el pico). En la mayoría de piezas con cámara globular el asa se encontraba sobre la cámara, en cambio en aquellas escultóricas la vertedera era colocada en el lado posterior del cuerpo (figs. 1a y b). La creación de la vertedera era un momento crucial en el proceso de fabricación de estas vasijas, debido a que, además del simbolismo inherente a su forma y decoración (Bock 2012: 22-24), el asa estribo permitía sostener a la pieza durante su manipulación y transporte. Las botellas eran elementos esenciales en la negociación de identidades mediante encuentros sociales públicos y privados, siendo empleadas en eventos ceremoniales identificados en contextos arqueológicos y representaciones cerámicas. Estas últimas muestran el uso de botellas de asa estribo en procesiones, producción textil, festines, presentación de vasos de libación y ritos funerarios (Benson 1975: Figuras 1, 12, 29; Donnan 1978: Figuras 10, 103, 2004 : Figura 1.17; Hocquenghem 1987: Figuras 14, 34; Donnan \& McClelland 1999: Figuras 1.13, 1.14, 1.15, 4.50, 4.78, $4.95,6.42$, 6.84) (figs. 2a-c). ${ }^{1}$ Las evidencias iconográficas y contextuales indican que las botellas cerámicas devinieron en bienes cargados de simbolismo religioso y en objetos estrechamente vinculados a la legitimación de la autoridad, la competencia por estatus y el acceso a redes de distribución de artículos de prestigio.

Los aspectos tecnológicos de la producción de cerámica Moche empezaron a ser objeto de interés académico décadas después que las vasijas del Estilo Moche se convirtieran en una de las principales fuentes de datos sobre la ideología de esta sociedad prehispánica andina. A pesar de su aparición relativamente tardía, el estudio de la elaboración de cerámica es en la actualidad un prometedor campo de trabajo, cuyos resultados demuestran la variabilidad tecnológica y la carga simbólica existentes al interior de la producción artesanal del norte peruano del primer milenio DC

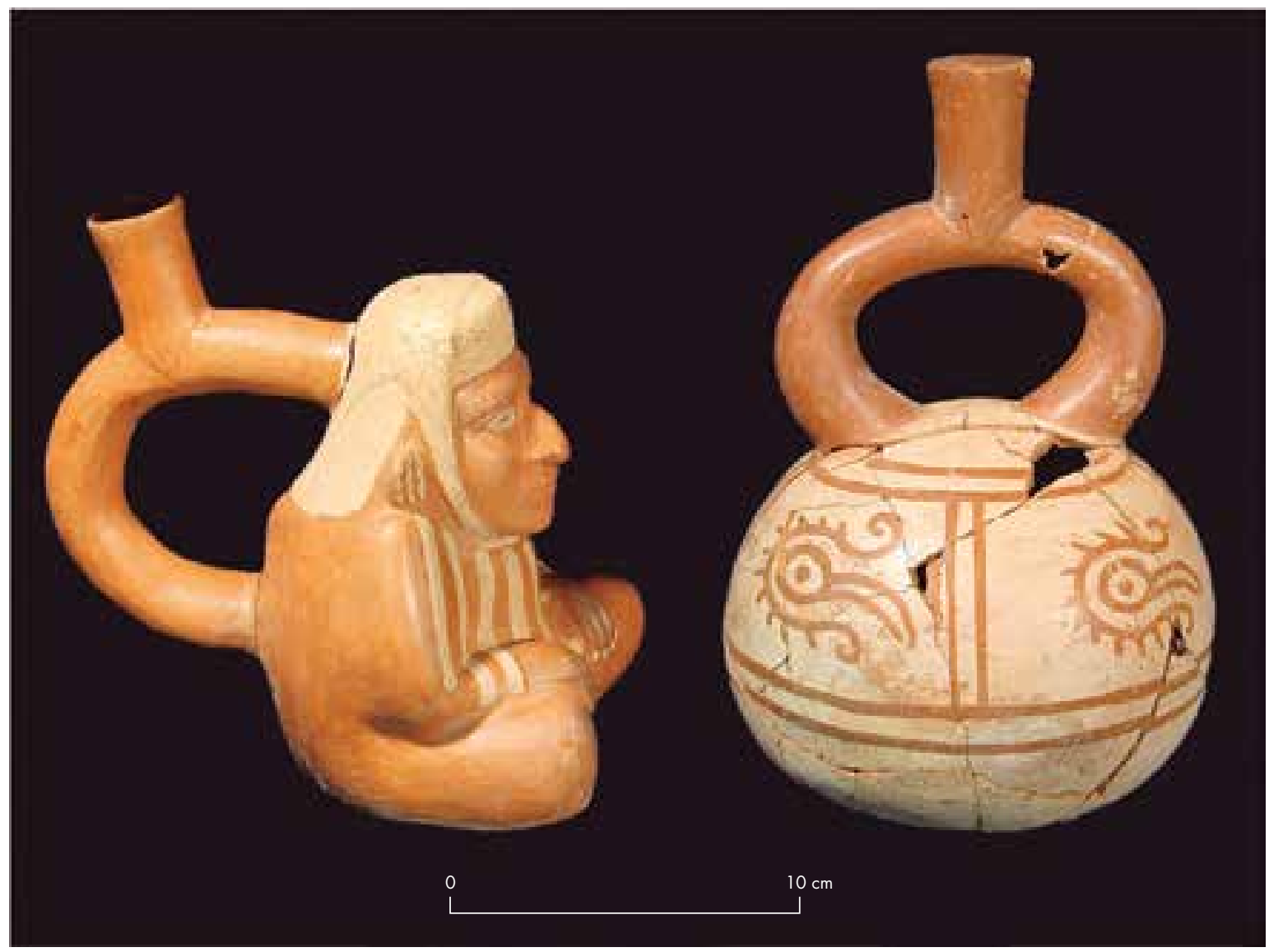

Figura 1. Vasijas de Tumba 1-PSUM 2000. a) Botella EC-36; b) Botella EC-37. Fotos: Claude Chapdelaine. Figure 1. Vessels from Tomb 1-PSUM 2000. a) Bottle EC-36; b) Bottle EC-37. Photos: Claude Chapdelaine. 
a
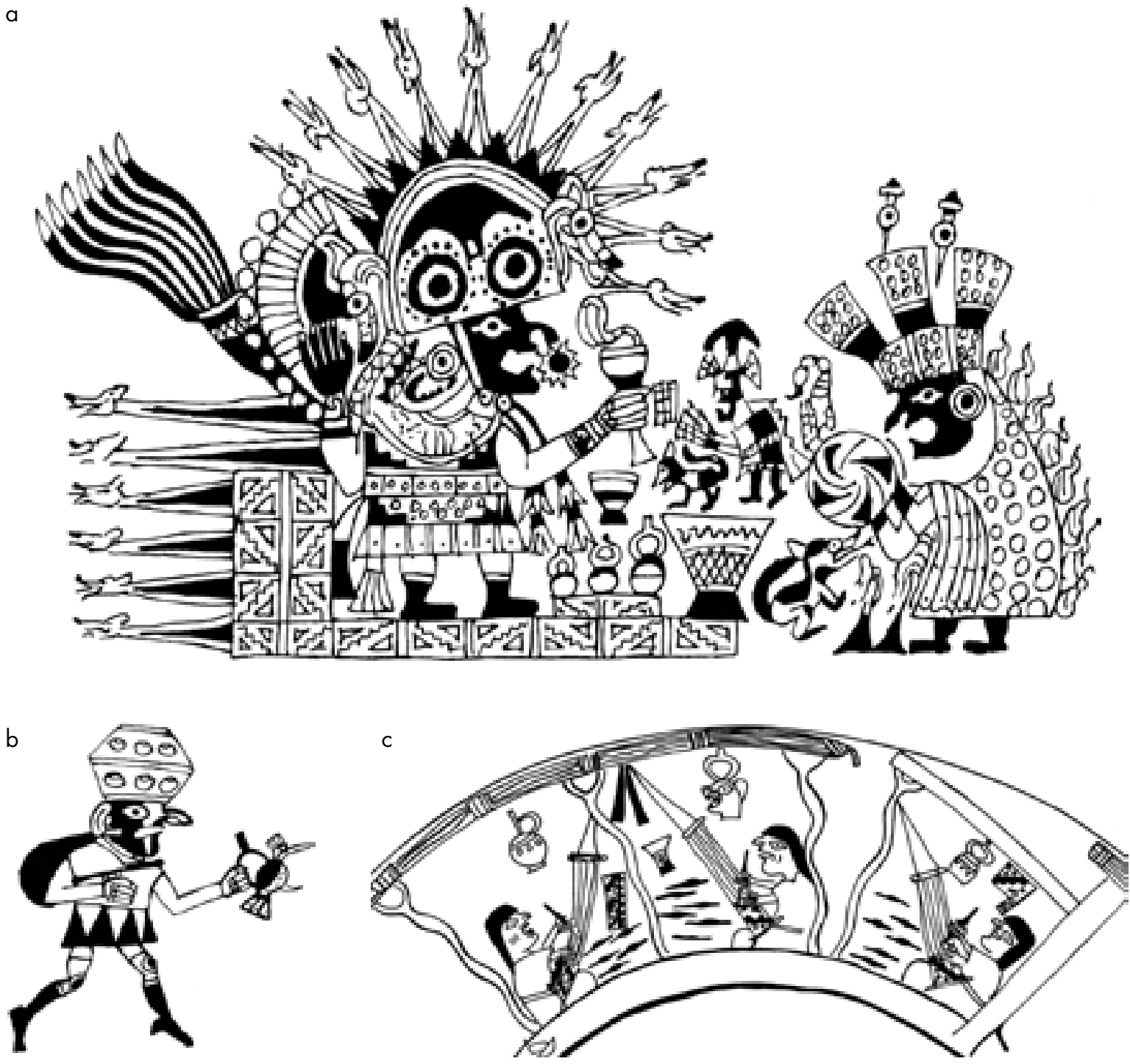

Figura 2. Representaciones en línea fina del empleo de botellas de asa estribo: a) Escena de presentación de copas (adaptado de Benson 1982: Figura 11); b) Personaje sosteniendo botella en la mano (adaptado de Donnan \& McClelland 1999: Figura 6.84); c) Escena de producción textil (adaptado de Donnan 1978: Figura 103).

Figure 2. Fine-line drawings from stirrup spout bottles: a) Raised cup scene (adapted from Benson 1982: Figure 11); b) Figure bolding a bottle (adapted from Donnan \& McClelland 1999: Figure 6.84); c) Textile production scene (adapted from Donnan 1978: Figure 103).

(Bernier 2008; Fraresso 2010). Este artículo presenta los primeros resultados del análisis de botellas del estilo cerámico Moche III procedentes del sitio El Castillo de Santa. El estudio de estos materiales permitió identificar una técnica de elaboración de botellas caracterizada por la creación de la vertedera mediante la unión progresiva de segmentos tubulares, modalidad que se diferencia claramente de la forma de fabricación de asas estribo Moche hasta ahora conocida.
ESTILOS CERÁMICOS, CRONOLOGÍA Y PROCESOS SOCIOPOLITICOS MOCHE EN SANTA

Las investigaciones realizadas en el valle de Santa (Donnan 1973; Wilson 1988; Chapdelaine 2008, 2010) han revelado la existencia de numerosos sitios Moche con materiales cerámicos pertenecientes a las Fases III y IV en la secuencia propuesta por Rafael Larco (1948). Al 
respecto, debe señalarse que si bien el esquema de Larco continúa siendo una herramienta válida en la definición de la cronología del territorio meridional Moche (Castillo \& Donnan 1994: 157-159), el incremento de dataciones radiométricas ha llevado a reconocer que las "fases" corresponden a variantes estilísticas cuya duración, variabilidad interna e interrelaciones mutuas pudieron diferir localmente. Bajo esta perspectiva, el registro de la distribución de estas unidades estilísticas es parte integral del estudio de los factores socioculturales con los cuales la evolución de las tradiciones artesanales y tecnológicas se vio entrelazada.

Nuestro estudio estará enfocado en el valle bajo de Santa, el que hacia 300 DC pasó a formar parte de una extensa esfera de interacción regional que integraba a los valles ubicados entre Chicama y Huarmey (Castillo \& Uceda 2008: 715-718; Chapdelaine et al. 2009a; Makowski 2009). A diferencia de la mayoría de los ríos de la costa peruana, el río Santa conduce agua a lo largo de todo el año, permitiendo una producción agrícola casi continua, crucial para las sociedades locales y los grupos foráneos que a lo largo del tiempo han interactuado con los "santeños". 2

La ocupación Moche en Santa fue evaluada a partir del año 2000 por el Proyecto Santa de la Universidad de Montreal (PSUM) (Chapdelaine et al. 2009a, 2009b; Chapdelaine 2010). Las excavaciones en el sitio El Castillo de Santa revelaron una notable concentración de arquitectura pública y residencial datada entre 300 y 600 DC y con cultura material y fechados comparables a los del Período Moche III y la transición Moche III/IV en el sitio de Huacas de Moche (Chapdelaine et al. 2001: 370-371, 2009a: 194-195, 198-200; Chapdelaine \& Pimentel 2003; Uceda et al. 2008). ${ }^{3}$ El centro Moche en El Castillo de Santa coexistió con una serie de entidades sociopolíticas del valle medio y alto de Santa afiliadas a las manifestaciones culturales Gallinazo y Recuay (Chapdelaine et al. 2009a: 195-201), constituyéndose en la sede de un linaje acaso originado en los valles de Moche o Chicama, pero cuyo poder fue consolidado, y eventualmente retado, por medio del establecimiento de alianzas y relaciones de competencia con otros asentamientos locales (fig. 3). Los niveles estratigráficos tempranos y medios de los sectores excavados presentaron como elementos cerámicos diagnósticos a las botellas de asa estribo con pico acampanulado, los cuencos de borde expandido (flaring bowls) con aplicaciones escultóricas sobre el labio y los cuencos de perfil compuesto o de borde reforzado, formas que muestran rasgos característicos de lo que ha sido denominado fase o subestilo Moche III (Donnan \& McClelland 1999: 47-73; Chapdelaine et al. 2001: 365-368; Donnan 2011: 117).
Otros trabajos del PSUM evidenciaron una nueva reestructuración del valle entre 600 y 700 DC, proceso que incluyó el abandono progresivo de El Castillo de Santa, la construcción de un nuevo asentamiento principal en Guadalupito y la ampliación de la frontera agrícola mediante la irrigación del sector Lacramarca (Chapdelaine \& Pimentel 2002; Gamboa 2008; Chapdelaine et al. 2009a: 201-203). Estos cambios fueron acompañados por la aparición en sitios habitacionales, sitios administrativos y centros ceremoniales de vasijas del Estilo Moche IV con formas y motivos similares a los reportados en los valles de Moche y Chicama (Uceda \& Armas 1998; Donnan \& McClelland 1999: 82-137; Gumerman \& Briceño 2003: 235; Donnan 2011: 116-117). Para este período es evidente el surgimiento en el valle costeño de Santa de un ethos Moche local, desarrollado en paralelo a una integración política y económica con el valle de Moche (Chapdelaine 2008, 2010; Chapdelaine et al. 2009a, 2009b; ver también Quilter 2010: 237). El período terminal Moche IV (700-750 DC) en Santa correspondió a poblaciones que precedieron o fueron coetáneas a la formación del Estilo Tanguche Temprano (Wilson 1988; Chapdelaine et al. 2004; Belisle 2008). Este tiempo estuvo marcado por profundas transformaciones en el paisaje urbano de Guadalupito, el abandono de Lacramarca y la introducción de cerámica Moche $\mathrm{V}$ en los estilos de Galindo del valle de Moche (Chapdelaine \& Pimentel 2001: 16; Pimentel \& Paredes 2003: 285-295) y Moro de Jequetepeque (Wilson 1988: Figura 234.V).

\section{La muestra cerámica de El Castillo de Santa}

Las excavaciones del PSUM (años 2000 a 2005) en los lados norte y este de El Castillo de Santa condujeron al registro de una amplia muestra de cerámica Moche procedente de rellenos arquitectónicos, pisos, tumbas y depósitos de ofrendas (Chapdelaine \& Pimentel 2001, 2002; Chapdelaine et al. 2005). La ladera septentrional del sitio presentaba una plataforma de adobe decorada con murales (Wilson 1988: 211) y un sector lateral denominado Terraza Norte, este último comprendiendo un sistema de patios y recintos conectados mediante rampas. La Terraza Norte conserva evidencias del cumplimiento de ceremonias comunitarias, rituales funerarios y actos dedicatorios, con una compleja secuencia de renovación arquitectónica atestiguada por la superposición de 3 a $5 \mathrm{~m}$ de estructuras y rellenos constructivos. En las áreas excavadas no se registraron hornos, concentraciones de cerámica cruda o sobrecocida u otros rasgos que señalaran la presencia de espacios de producción de vasijas en ese sector del asentamiento. 


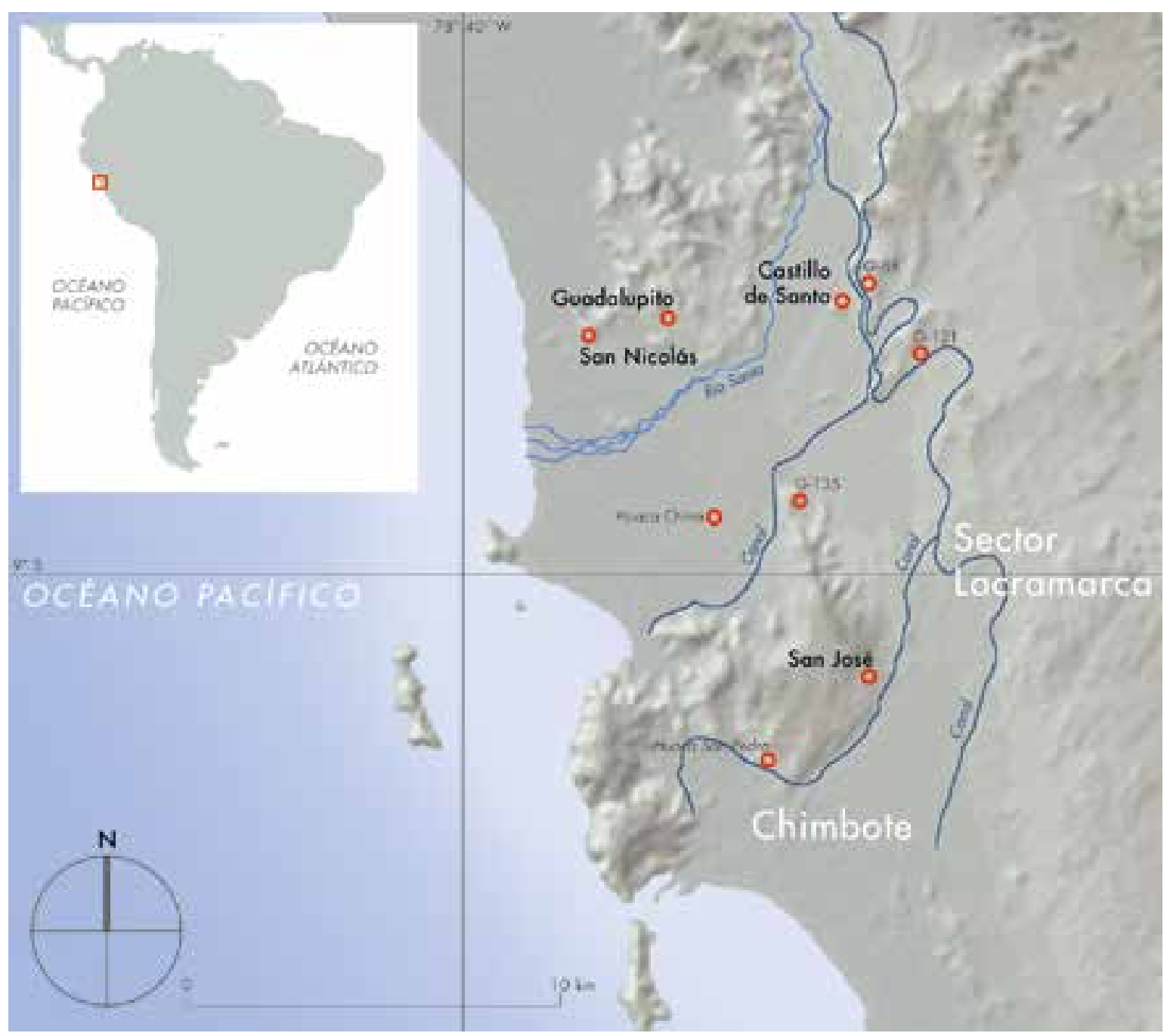

Figura 3. Sitios principales de ocupación Moche en el valle bajo de Santa.

Figure 3. Principal Moche occupational sites in the lower Santa Valley.

El conjunto analizado de botellas de Terraza Norte estuvo compuesto por 64 fragmentos de asas estribo procedentes de distintos niveles de pisos y rellenos y dos ejemplares completos documentados en un entierro. ${ }^{4} \mathrm{La}$ mayoría de las piezas fueron quemadas en hornos abiertos, presentando pastas de tonalidad naranja o rojiza. Estos materiales forman parte de un conjunto más extenso de cerámica fina y llana Moche documentada por el PSUM y analizada por el autor durante los últimos años.

\section{ESTUDIOS PREVIOS SOBRE LA ELABORACIÓN DE BOTELLAS MOCHE}

Uno de los primeros tratados sobre la elaboración de las botellas Moche fue desarrollado en 1939 por Adrian
Digby, trabajo realizado sobre un lote de vasijas en el British Museum que marcaría el inicio de la aplicación de la radiografía al estudio de la cerámica prehispánica peruana. Partiendo de una referencia proporcionada por Alfred Kroeber, Digby (1948: 607-608, 1952: 114) logró identificar la formación de algunas vertederas con base en tubos colocados sobre las cámaras, y propuso una evolución tecnológica de las botellas del norte peruano que se inicia con piezas de asas insertadas en las cámaras, luego se reemplaza por vasijas con asas estribo adheridas a la vasija y, finalmente culmina en la fabricación en un solo molde de asas y cámaras. Digby (1948: 607) denominó a las asas estribo Moche como four-piece spouts, pero incluyó bajo esa definición tanto a piezas con arcos elaborados mediante tubos acoplados como a ejemplares con asas cortadas, las 
que serían mejor caracterizadas dos décadas después. Otra referencia "temprana" a la elaboración de botellas Moche fue proporcionada por Lee A. Parsons (1962: 516-517), quien examinó un grupo de piezas Moche III con cámaras morfológicamente similares. Basándose en las observaciones de Digby, Parsons advirtió que los artesanos Moche mostraban preferencia por adherir los extremos del asa a la pared de la cámara en lugar de insertarlos en ella.

En la década de 1960, Christopher Donnan (1965, 1992: 60-62, 2004: 30-31) analizaría la cerámica funeraria excavada por Max Uhle en Huacas de Moche. A partir del estudio de esa colección, Donnan identificó el empleo conjunto de moldeado, modelado y enrollado para crear las partes de una botella. La forma de elaboración de asas estribo identificada por Donnan (1965: 123, Láminas II y III) puede ser denominada técnica del arco cortado, y empezaba con la creación sobre varillas de madera de los tubos que formarían el arco y el pico. La unión de esos segmentos daba origen a un asa incipiente, manipulada en pasta fresca hasta conseguir la curvatura y la posición del pico deseadas por el ceramista (Donnan 1965: Figuras 17-22). El asa estribo ya completa era adherida a hoyos recortados en la cámara, con rolletes de arcilla recubriendo el ángulo exterior de cada empalme. El paso siguiente estaba dedicado a reforzar internamente la unión del arco al cuerpo de la vasija y se realizaba sobre una botella completa, pero de pasta aún maleable. El alfarero efectuaba un corte en el eje sagital del arco tubular e introducía un alisador delgado, con cuyo extremo ejercía presión sobre el área de contacto entre el asa y la cámara. El corte era posteriormente cerrado y alisado.

Sergio Purin $(1983,1985)$ examinaría la propuesta de Donnan a partir del estudio de vasijas Moche presentes en los Musées Royaux d'Art et d'Histoire de Bélgica. Utilizando placas radiográficas, Purin detectó las irregularidades al interior del asa originadas por el trabajo de corte y cerrado del arco tubular, localizando las áreas de corte en ambos lados de asas colocadas en la parte superior de la botella (Purin 1983: Lámina V-B), en el lado más largo de asas estribo laterales (Purin 1983: Lámina II-A, B, Lámina III-A, Lámina IV-A y Lámina V-F), o en ambos lados de asas laterales (Purin 1985: 96-97, 100-101). La existencia de dos puntos de corte y cerrado condujo a Purin (1983: 11-12) a indicar que la base del pico podía ser dispuesta en un tercer orificio, con los picos de las botellas de asa estribo lateral siendo colocados luego de la unión del arco a la cámara. Varias de las piezas estudiadas pertenecían a las fases Moche I y Moche II, mostrando la aparición temprana de la técnica del asa cortada (Purin 1983: 7, 9). Purin también sugeriría que los alisadores introducidos en las asas podían ser flexibles y que el arco era formado a partir de un solo tubo alargado.

Hasta la década de 1980, el estudio de la fabricación de las botellas Moche había conducido a un conocimiento bastante detallado de la fabricación de esas piezas. Sin embargo faltaba reconocer si la producción de las vasijas más emblemáticas de la cultura Moche podía incluir procedimientos diferentes a los hasta entonces definidos.

\section{BOTELLAS CON ASAS ESTRIBO ELABORADAS MEDIANTE TUBOS INSERTADOS}

\section{La cadena operativa identificada}

El estudio de la producción de botellas Moche III a partir de las huellas de fabricación presentes en las propias piezas (Pernot 2010: 334) adquiere especial valor si consideramos que aún se carece de datos sobre los espacios de producción (así como de concentraciones de desechos y subproductos de la elaboración) de esos artefactos. La secuencia operacional de elaboración de botellas de asa estribo aquí propuesta fue reconstruida mediante análisis visual y exámenes radiográficos complementarios de especímenes procedentes de la Terraza Norte de El Castillo de Santa (figs. 4 a y b), una aproximación que permitió identificar tanto la cadena de gestos técnicos seguida por los artesanos como los tipos de herramientas empleadas y su momento de uso dentro del proceso de fabricación. El análisis permitió observar una técnica de construcción caracterizada por la manufactura del asa mediante el acoplamiento de tubos de distinta longitud. Treinta y ocho ejemplares (57\% del total de la muestra) presentaron esta modalidad, con grupos proporcionalmente similares de asas colocadas en forma lateral o dispuestas sobre cámaras globulares.

\section{La secuencia de elaboración}

1. Elaboración de la cámara. La elaboración de la cámara mediante el uso de moldes era el primer paso en la creación de las botellas, con el tamaño y la forma de esa parte de la vasija contribuyendo a determinar la dimensión, la curvatura y la posición del asa estribo. Al término de la formación de la cámara se recortaban en ella dos perforaciones circulares (fig. 5a). Estos hoyos eran situados en la parte superior (en piezas de cuerpo globular y en algunas escultóricas) o en el lado posterior de la vasija (en piezas escultóricas complejas). 

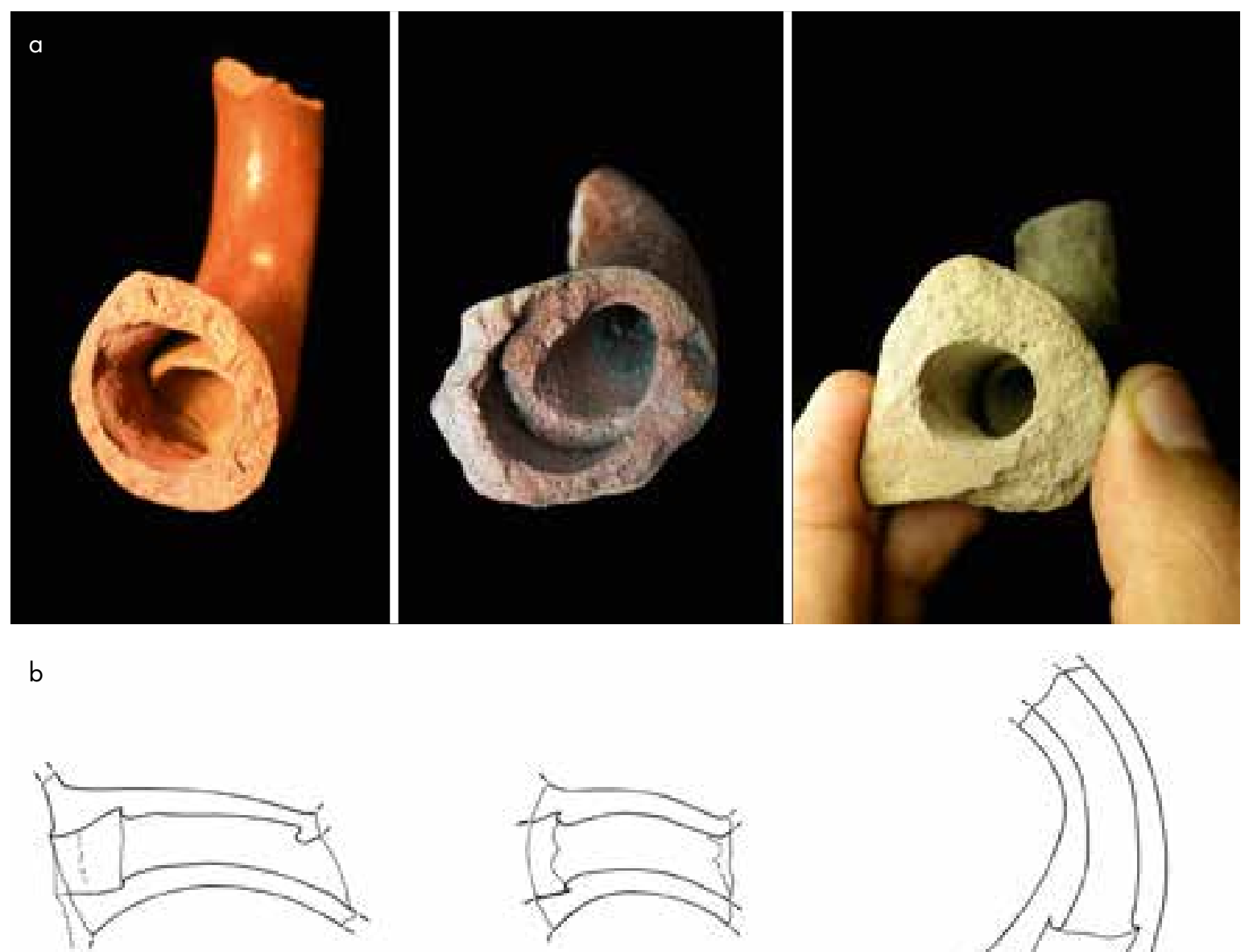

EC-7541

EC-4 152

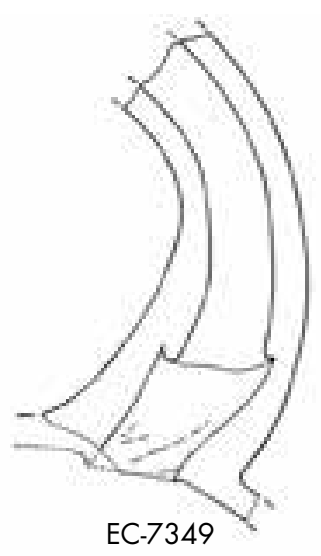

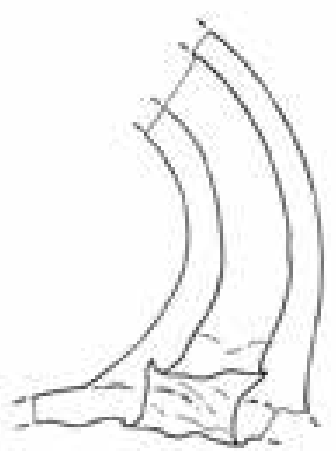

EC-7132

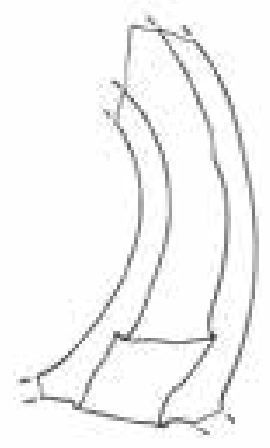

EC-8405

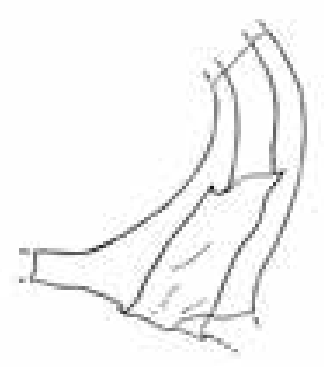

EC-4463

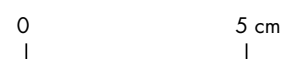

Figura 4. Asas estribo elaboradas mediante unión de segmentos tubulares registradas en El Castillo de Santa. a) Tubos base. Especímenes EC-4231, EC-7466 y EC-7349; b) Secciones elaboradas a base de radiografías. Fotos y dibujos: Jorge Gamboa.

Figure 4. Stirrup spouts made by joining together tubular ceramic segments, recorded at El Castillo de Santa. a) Base tubes. Items EC-4231, EC-7466 and EC-7349; b) Cross-sections based on x-ray analysis. Photos and drawings: Jorge Gamboa. 


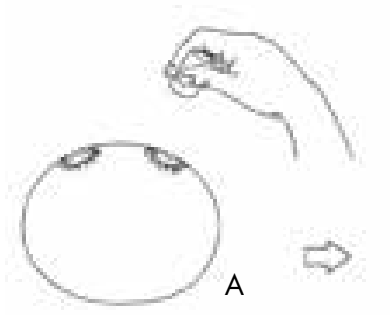

A
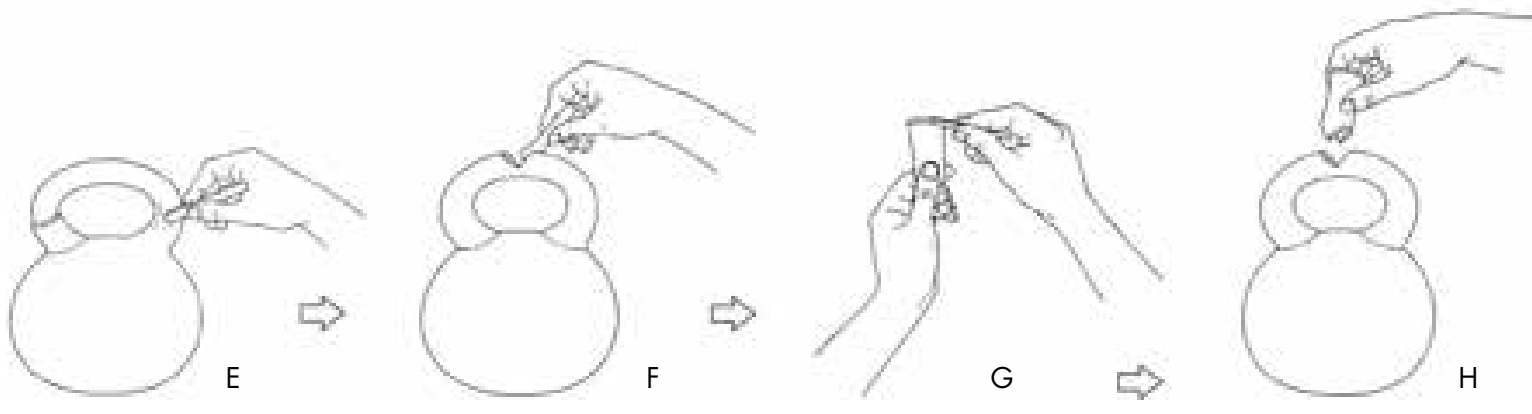

Figura 5. Proceso de construcción de botellas Moche III con asa estribo elaborada mediante segmentos tubulares insertados. Dibujos: Jorge Gamboa.

Figure 5. Process used to construct Moche III stirrup spout bottles by inserting tubular segments. Drawings: Jorge Gamboa.

2. Formación de tubos. Empleando varillas de madera como núcleos, el artesano elaboraba luego cuatro tubos de arcilla destinados a formar el pico, el segmento central del arco, y un par de cilindros cortos (a los que en adelante denominaremos tubos base). El último par de piezas tenía un diámetro ligeramente mayor a los segmentos restantes, indicando su elaboración sobre varillas más gruesas.

3. Colocación de los tubos base. La etapa inicial en la construcción del asa estribo consistía en la colocación (en ángulo recto u oblicuo con el extremo inferior cortado en forma diagonal) de la primera pareja de tubos sobre la cámara. Los tubos base identificados en la muestra presentaron un diámetro promedio de $25 \mathrm{~mm}$, con los ejemplares más grandes alcanzando los $30 \mathrm{~mm}$. La unión de los tubos base al cuerpo de la vasija era reforzada exteriormente con un rollo de arcilla, cuyo alisado otorgaba mayor grosor y apariencia cónica a esas piezas tubulares (fig. 5b). Existían dos formas de colocación de los tubos base: a) Adherencia a la superficie de la cámara, realizándose pequeñas incisiones en el borde del tubo y alrededor de la perforación para consolidar la unión entre ambas partes. El ceramista alisaba el empalme ejerciendo presión con un alisador o introduciendo un dedo al interior del tubo (fig. 5c); b) Inserción en los orificios de la cámara, procedimiento que incluía la ejecución de incisiones verticales sobre la superficie exterior del tubo.

4. Formación del arco. El siguiente paso correspondía a la colocación del tercer segmento tubular (fig. 5d), una pieza previamente curvada cuyos extremos podían ser aplastados o cortados en forma diagonal para facilitar su inserción en los tubos base. La disposición del segmento central completaba la creación del arco, el que comprendía cuatro puntos de unión regularizados mediante agregado de pasta húmeda y alisado (fig. $5 \mathrm{e}$ ).

5. Colocación del pico. La adición del pico era realizada insertando la base de un cuarto tubo en un recorte en $\mathrm{V}$ ejecutado en el arco (figs. 5f-h). La base del pico era levemente aplastada antes de ser introducida, quizás para corregir diferencias de diámetro con el orificio en el asa. La unión interior entre estas partes era alisada con un punzón delgado. La base del gollete solía conservar al exterior la forma del corte en el arco.

Los ceramistas finalizaban su obra aplicando engobes crema o rojos sobre la vasija, procediendo a pulirla antes de su secado completo y quemado. El color del engobe elegido para el asa solía contrastar con el de la cámara. En la muestra de botellas Moche III de El Castillo de Santa no se identificaron asas estribo con decoración en línea fina, sin embargo se registró el uso 
de pintura negra orgánica aplicada sobre el pico o en el arco tubular.

\section{BOTELLAS CON ASAS ESTRIBO CORTADAS}

Veintiséis asas fragmentadas y dos especímenes completos de la muestra cerámica de Terraza Norte presentaron la técnica del arco cortado. Dentro de este grupo la cantidad de asas estribo laterales fue similar a la de aquellas colocadas en la parte superior de las vasijas, observándose mayoritariamente en ambos grupos la adherencia del arco a la superficie exterior de la cámara. La información más relevante sobre este tipo de asas procede de las botellas completas registradas en la Tumba 1-PSUM 2000 , un contexto funerario localizado junto a un muro monumental de adobe (Chapdelaine et al. 2005: 20-23).

La primera botella (EC-36), ubicada a la derecha del infante sepultado en la tumba, representaba a un hombre ataviado con camiseta, tocado textil y orejeras (fig. 1a). En la radiografía se observó la unión vertical de las mitades moldeadas de la cámara y el cierre

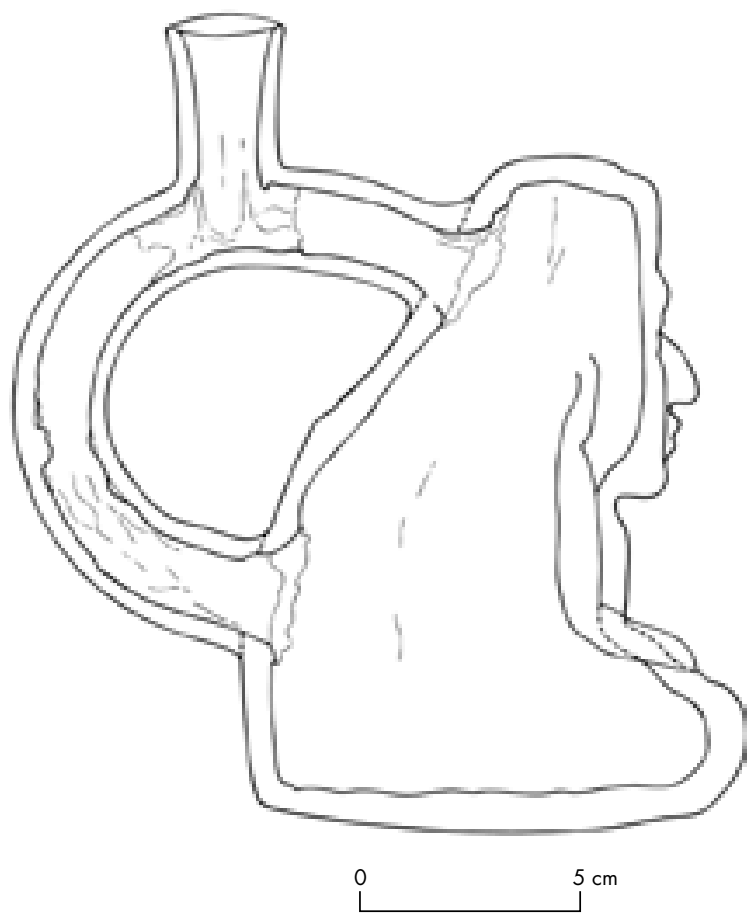

Figura 6. Sección basada en radiografía de botella de asa estribo (EC-36) de Tumba 1-PSUM 2000. Dibujo: Jorge Gamboa.

Figure 6. Cross-section of a stirrup spout bottle (EC-36) recovered from Tomb 1-PSUM 2000, based on x-ray analysis. Drawing: Jorge Gamboa.

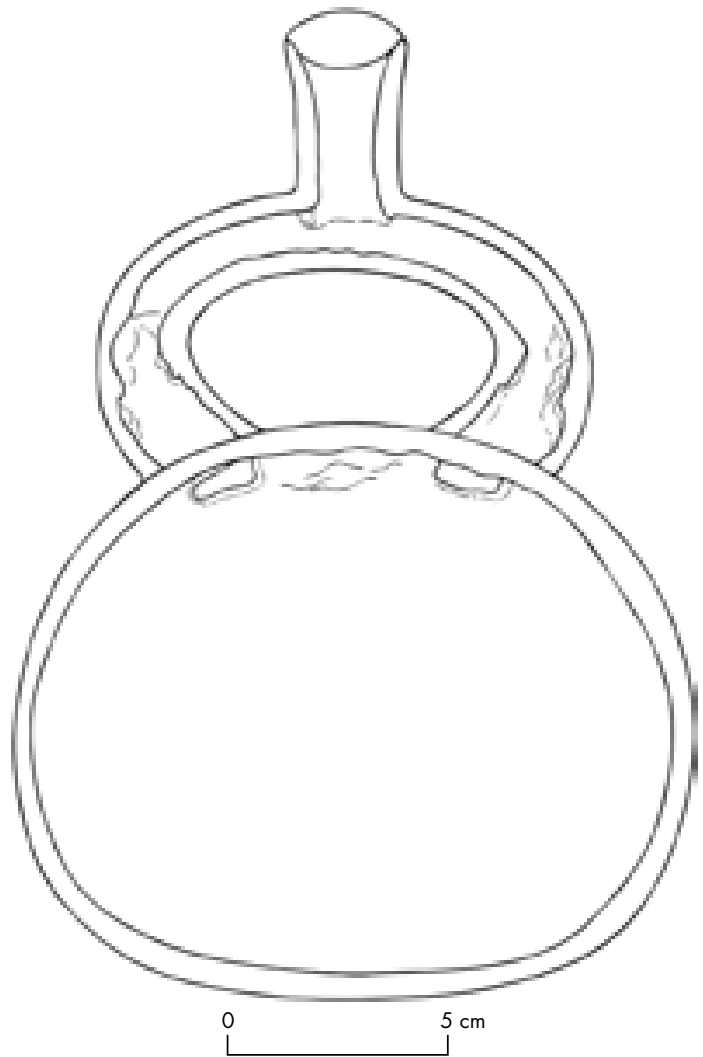

Figura 7. Sección basada en radiografía de botella de asa estribo (EC-37) de Tumba 1-PSUM 2000. Dibujo: Jorge Gamboa.

Figure 7. Cross-section of a stirrup spout bottle (EC-37) from Tomb 1-PSUM 2000, based on x-ray analysis. Drawing: Jorge Gamboa.

manual de la base de la vasija (fig. 6). La mitad inferior del asa estribo presentó un corte de $2,5 \mathrm{~cm}$, usado para alisar la unión interior del arco a la cámara. La mitad superior del arco conservaba trazas del alisado interno, paso que debió ser realizado por el orificio empleado para colocar el pico. El segundo ejemplar (EC-37) fue hallado a la izquierda del niño y presentaba cámara globular decorada con diseños pintados de cabezas de ave (fig. 1b). La placa radiográfica reveló que el cuerpo había sido elaborado en un molde bivalvo con abertura en la parte superior, espacio que fue cerrado manualmente mediante enrollado en espiral (fig. 7). En los hombros del arco se detectaron cortes de $2 \mathrm{~cm}$ de largo, frente a los cuales aparece un engrosamiento angular de la pared.

Las radiografías de otros ejemplares fragmentados pertenecientes a botellas escultóricas constataron las observaciones de Purin (1985: 101) sobre asas laterales con más de dos puntos de corte del arco tubular (figs. 8 y 9). La existencia de variantes en el número y 


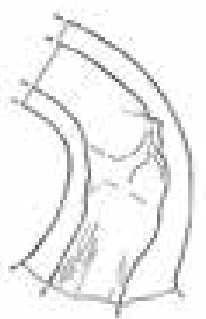

EC-4555

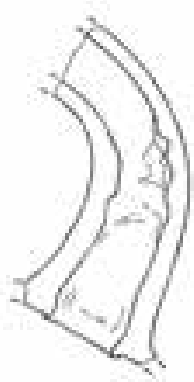

EC-5455
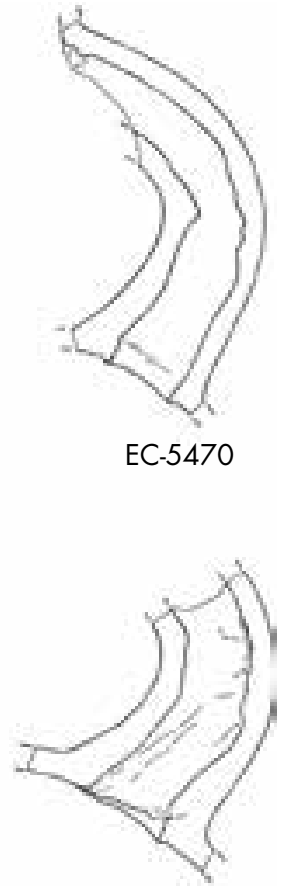

EC-5551

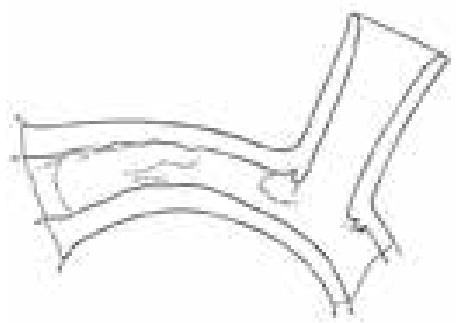

EC-45 11

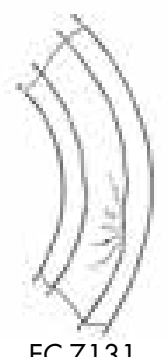

EC-7131

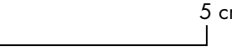

Figura 8. Secciones de asas estribo de Terraza Norte producidas mediante cortado del arco. Dibujos: Jorge Gamboa.

Figure 8. Cross sections of stirrup spouts from Terraza Norte, produced by cutting the arc. Drawings: Jorge Gamboa.

la posición de los cortes indicaría que su ejecución era producto de la combinación de dos factores: el tamaño y la curvatura del asa y el criterio individual del artesano sobre el punto correcto de colocación del pico.

\section{COMPARANDO CADENAS DE PROCEDIMIENTOS}

La comparación de las dos secuencias operacionales de construcción de botellas de asa estribo identificadas en El Castillo de Santa permite apreciar procedimientos diferenciados en la naturaleza y sucesión de las etapas del proceso de manufactura (fig. 10). Ambas técnicas de elaboración se iniciaban de forma similar, distinguiéndose marcadamente durante la creación manual de las asas. A pesar de ello, el resultado material de ambos procedimientos era la producción de objetos formalmente semejantes y portadores en común de los símbolos de la ideología Moche.

Las diferencias en los procesos de fabricación de las botellas de asa estribo aparecen concentradas en los momentos de la cadena operativa dedicados a la construcción del asa estribo. En la variante basada en la inserción de segmentos tubulares se enfatizaba el acoplamiento manual de las partes del arco y el alisado exterior de los puntos de unión. En cambio, poner en práctica la técnica del asa cortada implicaba el uso de un artefacto cortante, alisadores simples y un alisador alargado y tal vez flexible, herramientas necesarias para abrir la pared del arco y consolidar interna y externamente su unión con la cámara.

Los artesanos especializados en la técnica de tubos insertados realizaban la fabricación de las botellas por medio de la manipulación conjunta de cámaras y asas, solucionando el punto crítico de unión entre ambas partes en el momento inicial de manufactura del asa estribo, a diferencia de la formación por separado del cuerpo y el asa en las piezas elaboradas mediante la técnica del arco cortado. En ambos grupos de vasijas los pasos finales de acabado y decoración de superficies eran nuevamente semejantes. Queda por determinar si los ceramistas Moche podían recurrir libremente a cualquiera de las variantes, o si actuaban bajo reglas más estrictas dictadas por afiliación a determinados talleres o corporaciones laborales.

\section{COMENTARIOS FINALES}

A partir del análisis de la cerámica de El Castillo de Santa se han confirmado las referencias tempranas de Digby $(1948,1952)$ sobre la creación de asas estribo con tubos acoplados. ${ }^{5}$ Asimismo, se identificaron dos diferencias primarias de esta técnica frente a la del arco cortado: el empleo de un número mayor de tubos para formar el asa estribo, y la fabricación gradual del asa. La elaboración de botellas de asa estribo en la costa norte de Perú en los primeros siglos DC incorporó un alto grado de habilidad en el empleo de moldes, pastas y pigmentos, pero también una inversión adicional de tiempo y destreza por los ceramistas para culminar cada pieza. El modelado de las vertederas producía diferencias en la forma y el tamaño de esas partes de las vasijas, 

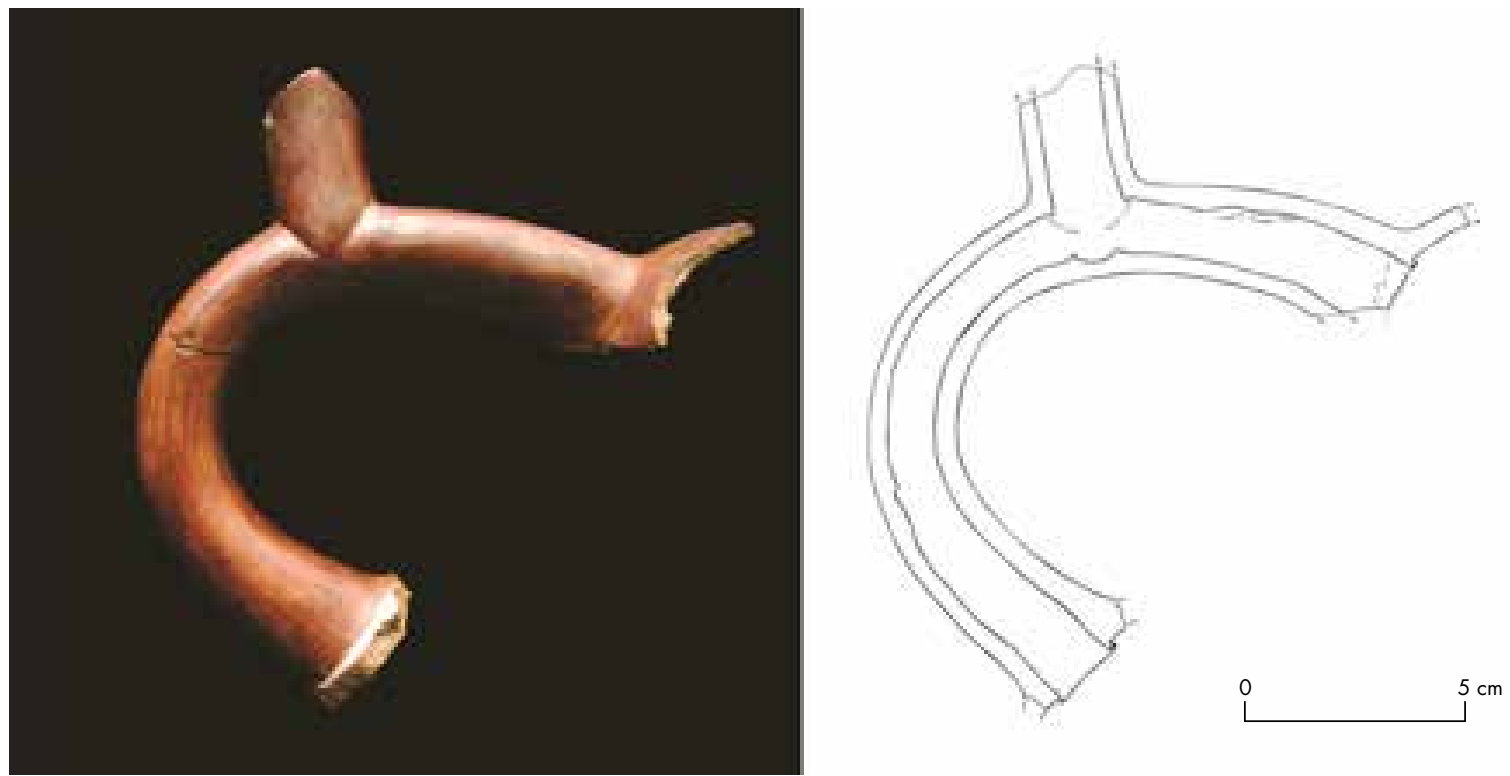

Figura 9. Fotografía y sección basada en radiografía de asa estribo EC-844 8. Foto y dibujo: Jorge Gamboa.

Figure 9. Photograph and cross-section of the stirrup spout EC-844 8, based on x-ray analysis. Photo and drawing: Jorge Gamboa.

aun en aquellas con cámaras elaboradas en moldes similares (ver Donnan 2004: 94-95, 100-103, 106-109). Nuestros datos permiten apreciar la complejidad de los procesos de construcción de las botellas Moche de asa estribo, en especial si se los compara con las técnicas popularizadas luego del 900 DC por los alfareros Chimú, quienes emplearían procedimientos más expeditivos de moldeado para obtener una vasija de asa estribo en una de las últimas innovaciones de la tradición cerámica regional después del prolífero tiempo de experimentación tecnológica y artística Moche.

La procedencia estratigráfica de la muestra tiende a confirmar que las botellas de asa estribo Moche fueron usadas -igual que otros tipos de vasijas decoradas- en un amplio conjunto de actividades rituales y cotidianas (Donnan \& McClelland 1999: 18-19; Bernier 2009: 166167). Los especímenes analizados fueron recuperados en rellenos arquitectónicos, pisos y tumbas, con los dos primeros grupos de procedencia, sugiriendo que la utilización de las piezas podía conducir a su eventual fragmentación y descarte junto a otros desechos. Como se señaló con anterioridad, las botellas de asa estribo habrían sido exhibidas y manipuladas durante diversos rituales y actividades festivas, con esas formas de uso debiendo ser consideradas complementarias a la disposición final de algunos ejemplares como ofrendas mortuorias (para un punto de vista opuesto ver Bourget 2006: 49).

En el asentamiento Moche de El Castillo de Santa se emplearon botellas elaboradas mediante las dos técnicas presentadas en este reporte. La pertenencia de ambos grupos de vasijas a una misma tradición alfarera es indicada por el empleo de pastas y engobes similares al nivel de observación macroscópica y por el predominio del quemado en hornos abiertos. Ambos conjuntos presentan semejanzas formales e iconográficas con la cerámica Moche III de los valles de Chicama (Franco et al. 2003: Lámina 19.3) y Moche (Chapdelaine et al. 2001: Figuras 5-8; Tufinio 2006: 38, 40; Tello \& Delabarde 2008: 151-155). Los resultados de nuestro análisis tienden a indicar que las similitudes externas entre las botellas de asa estribo iban emparejadas con variantes tecnológicas y operativas no destinadas a ser perceptibles por la mayoría de usuarios de estos bienes. La producción de objetos cerámicos exteriormente semejantes mediante cadenas operatorias distintas es en tal sentido comparable con la metalurgia Moche, cuyo complejo procedimiento técnico de recubrimiento con oro de piezas de cobre (p. ej. depletion gilding) revela un marcado interés en la apariencia y el color exterior de parafernalia ceremonial y ornamentos personales (Lechtman et al. 1982; Lechtman 1996). La complejidad en la elaboración de uno de los principales soportes cerámicos del arte y la ideología Moche parece indicar que el proceso de manufactura de botellas incorporaba lo que Cook (2004: 151) denomina "información cargada de valor" inherente tanto al objeto terminado como al proceso de su creación. La existencia de múltiples procedimientos técnicos por parte de los productores de cerámica y metales puede ser considerada en ese contexto un factor constitutivo tanto de la 
Técnica de segmentos tubulares insertados
Técnica de cortado del arco

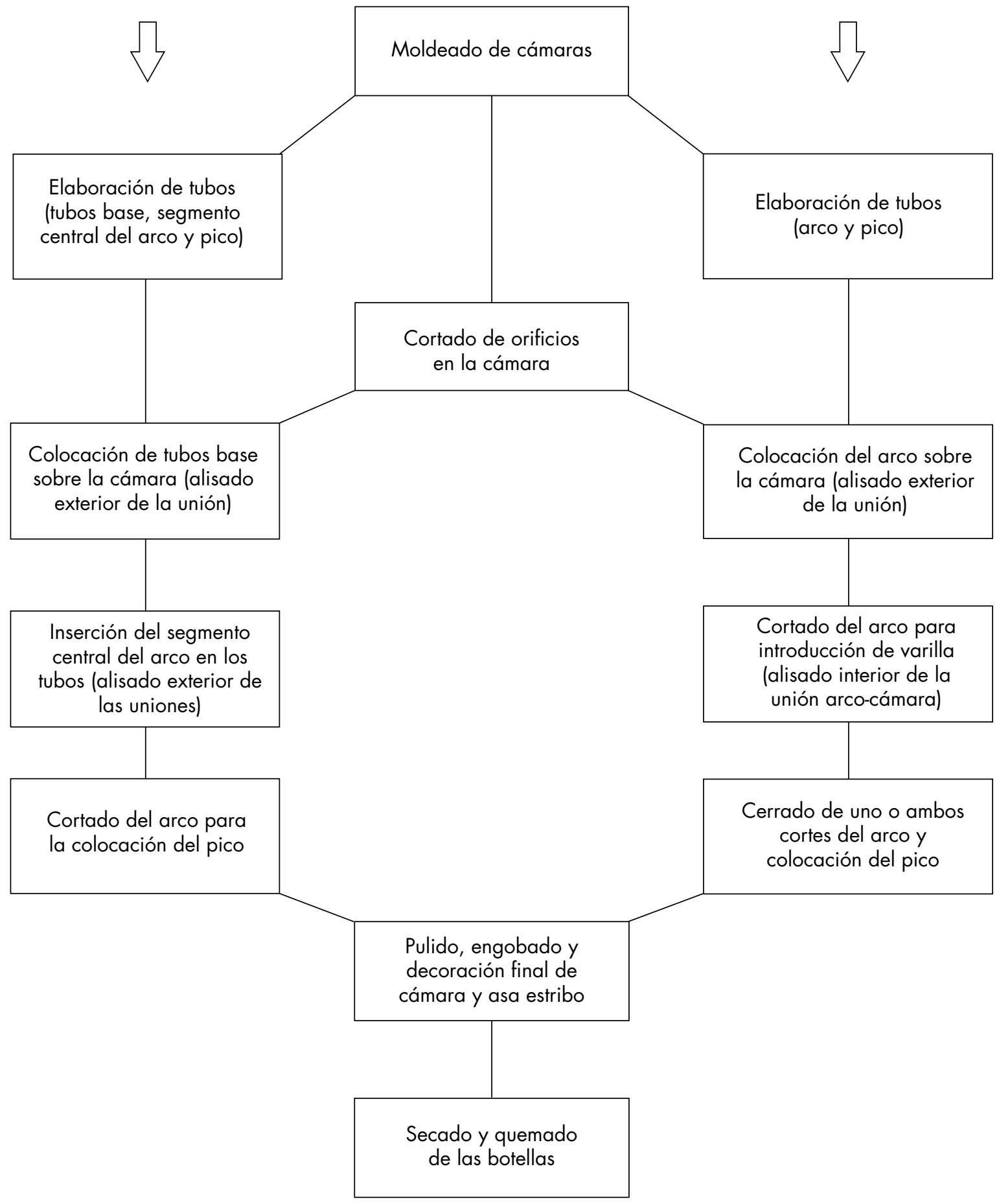

Figura 10. Cuadro comparativo de procesos de elaboración de botellas Moche de asa estribo.

Figure 10. Flowchart comparing processes for manufacturing Moche stirrup spout bottles. 
evolución de las tecnologías de producción como de la materialización de la ideología Moche.

A pesar de la importancia adquirida por estas piezas en los estudios iconográficos, aún no se han identificado talleres especializados en la elaboración de cerámica Moche III, lo que contrasta con la mejor conocida producción de vasijas y otros artefactos cerámicos Moche IV (Russell et al. 1998: 79-82; Uceda \& Armas 1998; Rohfritsch 2010: 391-395). La presencia de dos formas de elaboración de botellas Moche III en El Castillo de Santa indicaría que los ocupantes del sitio tuvieron acceso a la producción de talleres artesanales diferenciados por las preferencias técnicas de sus maestros ceramistas. ${ }^{6}$ Alternativamente, no puede descartarse que algunos espacios de producción hubieran reunido a alfareros trabajando bajo modalidades operativas distintas, pero que compartían un mismo bagaje de conocimientos tecnológicos y vocabularios visuales. El registro de espacios de producción cerámica Moche III deviene así en vital para determinar si el conocimiento actual sobre los talleres alfareros tardíos Moche refleja el carácter organizativo de la producción de cerámica más temprana.

Las investigaciones del PSUM muestran que la fabricación de asas con segmentos tubulares insertados también ocurre en la cerámica de Guadalupito, un asentamiento que hacia 600 DC desplazó a El Castillo de Santa como la capital del valle bajo de Santa. Debido al notable grado de interacción entre Santa y el área de Moche y Chicama durante los siglos IV a VIII DC, es factible sugerir que la elaboración de botellas de asas estribo formadas mediante tubos acoplados debe encontrarse también presente en las tradiciones alfareras de sitios como Huacas de Moche, El Brujo, Mocollope, Cerro Mayal, Licapa y Galindo. La posibilidad de hallar la modalidad de construcción de botellas identificada en El Castillo de Santa en otras entidades sociopolíticas Moche permanece abierta, debiendo ser confirmada con los resultados del análisis formal y tecnológico de la producción cerámica que realicen los proyectos dedicados a la investigación de estas sociedades prehispánicas del norte peruano.

RECONOCIMIENTOS Los trabajos de campo y gabinete del Proyecto Santa de la Universidad de Montreal fueron financiados por el Consejo de Investigaciones en Ciencias Humanas de Canadá. El presente artículo fue enriquecido en la Biblioteca de Estudios Precolombinos de Dumbarton Oaks durante el Summer Fellowship brindado al autor en el año 2009. Deseo expresar mi gratitud a Claude Chapdelaine y Víctor Pimentel Spissu, directores del PSUM, por su apoyo para la realización de este trabajo, así como a Hélène Bernier, Christopher Donnan, Régulo Franco, Sarahh Scher, Lisa Trever y Juliet Wiersema por sus valiosos comentarios y sugerencias. La digitalización de las imágenes se vio beneficiada en gran manera por el trabajo de Aldo Watanave. Igualmente se agradecen las observaciones y las sugerencias de tres revisores anónimos de una primera versión de este manuscrito.

\section{NOTAS}

1 Las reproducciones de escenas en línea fina que ilustran este artículo fueron elaboradas originalmente por Donna McClelland (Donnan 1978; Donnan \& McClelland 1999) y Gillett G. Griffin (Benson 1982). La figura 2a fue redibujada por Jorge Gamboa.

2 Donnan (1973) y Wilson (1988: 355) propusieron inicialmente la conquista de Santa por una entidad expansionista Moche III procedente del valle de Moche, idea que fue respaldada por la interpretación vigente hasta la década de 1990 de un dominio hegemónico regional del sitio Huacas de Moche.

3 Uceda y colaboradores (2008: 214-215, 221-222) señalan para Huacas de Moche la superposición temporal de los estilos Moche III y IV entre 450 y 550 DC, con los fechados posteriores a 600 DC procediendo de niveles estratigráficos asociados a materiales mayoritariamente Moche IV.

${ }^{4}$ Los fragmentos que componen la muestra fueron considerados correspondientes a diferentes botellas a partir de sus características de pasta, acabado y cocción.

5 Purin (1983: 11) señaló haber identificado una botella retrato con tubos cortos acoplados al segmento central del arco tubular.

6 Una primera fase de Análisis por Activación Neutrónica (AAN) de pastas cerámicas realizado por el PSUM incluyó 11 muestras de El Castillo de Santa, cinco de Guadalupito y seis de San José de Lacramarca. Las piezas de El Castillo de Santa no pertenecieron a un grupo composicional homogéneo, diferenciándose asimismo de otras vasijas del valle de Santa (Chapdelaine et al. 2009b: 57-64) y de Huacas de Moche (Chapdelaine et al. 1995) sometidas a AAN.

\section{REFERENCIAS}

Belisle, V., 2008. El Horizonte Medio en Santa. Continuidad y discontinuidad con los Mochicas del Intermedio Tardío. En Arqueología Mochica: Nuevos enfoques, L. J. Castillo, H. Bernier, G. Lockard \& J. Rucabado, Eds., pp. 17-31. Lima: Instituto Francés de Estudios Andinos y Fondo Editorial de la Pontificia Universidad Católica del Perú.

Benson, E., 1975. Death-associated figures on Mochica pottery. En Death and the Afterlife in Pre-Columbian America, E. Benson, Ed., pp. 105-144. Washington, D. C.: Dumbarton Oaks Research Library Collection.

— 1982. The Well-dressed Captives: Some Observations on Moche Iconography. Baessler-Archiv, Band XXX: 181-222.

Bernier, H., 2008. La especialización artesanal en el sitio Huacas de Moche: Contextos de producción y función sociopolítica. En Arqueología Mochica: Nuevos enfoques, L. J. Castillo, H. Bernier, G. Lockard \& J. Rucabado, Eds., pp. 33-51. Lima: Instituto Francés de Estudios Andinos y Fondo Editorial de la Pontificia Universidad Católica del Perú.

— 2009. La producción especializada de cerámica doméstica y ritual Mochica. Estudios Atacameños 39: 157-178.

Bock, E. DE, 2012. Sacrificios bumanos para el orden cósmico y la regeneración. Estructura y significado en la iconografia Moche. Trujillo: Ediciones Sian.

Bourget, S., 2006. Sex, Death and Sacrifice in Moche Religion and Visual Culture. Austin, TX: University of Texas Press.

Castillo, L. J. \& C. Donnan, 1994. Los mochicas del norte y los mochicas del sur. En Vicús, K. Makowski, Comp., pp. 143-181. Lima: Banco de Crédito del Perú.

Castillo, L. J. \& S. Uceda, 2008. The Mochicas. En Handbook of South American Archaeology, H. Silverman \& W. Isbell, Eds., pp. 707-729. New York: Springer.

Chapdelaine, C., 2003. La ciudad de Moche: Urbanismo y Estado. En Moche: Hacia el final del milenio, Tomo II, S. Uceda \& E. Mujica, Eds., pp. 243-285. Lima: Fondo Editorial de la Pontificia Universidad Católica del Perú. 
2008. Moche Art Style in the Santa Valley. Between Being "a la mode" and developing a Provincial Identity. En The Art and Archaeology of the Moche, S. Bourget \& K. Jones, Eds., pp. 129-152. Austin, TX: University of Texas Press

2010. Moche Political Organization in the Santa Valley: A Case of Direct Rule through Gradual Control of the Local Population. En New Perspectives on Moche Political Organization, J. Quilter \& L. J. Castillo, Eds. pp. 252-279. Washington, D. C.: Dumbarton Oaks Research Library and Collection.

Chapdelaine, C.; G. Kennedy \& S. UCedA, 1995. Activación neutrónica en el estudio de la producción local de cerámica ritual en el sitio Moche, Perú. Bulletin de l'Institut Français d'Études Andines 24 (2): 183-212, Lima.

Chapdelaine, C. \& V. Pimentel, 2001. Informe del Proyecto Arqueológico Santa de la Universidad de Montreal 2000. Lima: Instituto Nacional de Cultura.

— 2002. Informe del Proyecto Arqueológico Santa de la Universidad de Montreal 2001. Lima: Instituto Nacional de Cultura.

— 2003. Un tejido único Moche III del sitio Castillo de Santa: Una escena de cosecha de yuca. Bulletin de l'Institut Français d'Études Andines 32 (1): 23-50, Lima.

Chapdelaine, C.; V. Pimentel \& H. Bernier, 2001. A Glimpse at the Moche Phase III Occupation at the Huacas of Moche Site, Northern Peru. Antiquity 75: 361-372.

Chapdelaine, C.; V. Pimentel, G. Gagné, J. Gamboa, D. Regalado \& D. Chicoine, 2004. Nuevos datos sobre Huaca China, Perú. Bulletin de l'Institut Français d'Études Andines 33 (1): 55-80, Lima.

Chapdelaine, C.; V. Pimentel \& J. Gamboa, 2005. Entierros Moche en El Castillo de Santa. Una primera aproximación. En Corriente Arqueológica 1, C. Olaya \& M. Romero, Eds., pp. 13-41. Lima: Universidad Nacional Federico Villarreal.

_ 2009a. Gallinazo Cultural Identity in the Lower Santa Valley: Ceramics, Architecture, Burial Patterns, and Sociopolitical Organization. En Gallinazo. An Early Cultural Tradition on the Peruvian North Coast, J. F. Millaire \& M. Morlion, Eds., pp. 181-206. Los Angeles, CA: Cotsen Institute of Archaeology Press, University of California, Los Angeles.

- 2009b. Rol de la cerámica en la afirmación social de los Moches del Valle de Santa. Revista Chilena de Antropología 20: 37-76. Santiago: Universidad de Chile.

Cook, A., 2004. Wari Art and Society. En Andean Archaeology, H. Silverman, Ed., pp. 146-166. Malden, Oxford y Victoria: Blackwell Publishing Ltd.

DigBY, A., 1948. Radiographic Examination of Peruvian Pottery Techniques. En Actes $d u$ XXVIII Congrès International des Américanistes (1947), pp. 604-608, Paris.

- 1952. Three Cupisnique Vases in the British Museum. Man 52: $113-115$.

Donnan, C. B., 1965. Moche Ceramic Technology. Nawpa Pacha 3: 115-138. Berkeley: Institute of Andean Studies.

- 1973. Moche Occupation of the Santa Valley. Los Angeles, CA: University of California Press.

- 1978. Moche Art of Peru. Pre-Columbian Symbolic Communication. Los Angeles, CA: Museum of Cultural History, University of California.

- 1992. Ceramics of Ancient Peru. Los Angeles, CA: Fowler Museum of Cultural History.

— 2004. Moche Portraits from Ancient Peru. Austin, TX: University of Texas Press.

- 2011. Moche Substyles: Keys to Moche Understanding Moche Political Organization. Boletín del Museo Chileno de Arte Precolombino 16 (1): 105-118.

Donnan, C. B. \& D. MCClelland, 1999. Moche Fineline Paintings. Its Evolution and Its Artists. Los Angeles, CA: Fowler Museum of Cultural History.

Franco, R.; C. GÁlvez \& S. VÁsquez, 2003. Modelo, función y cronología de la Huaca Cao Viejo, Complejo El Brujo. En Moche: Hacia el final del milenio, Tomo II, S. Uceda \& E. Mujica, Eds. pp. 43-78. Lima: Fondo Editorial de la Pontificia Universidad Católica del Perú.
Fraresso, C., 2010. Estudio arqueométrico de un taller de transformación de cobre y de aleaciones tumbaga en el sitio de Huacas de Moche. Bulletin de l'Institut Français d'Études Andines 39 (2): 351-387, Lima.

GAmBOA, J., 2008. Plazas y cercaduras: Una aproximación a la arquitectura pública Moche IV y V en los valles de Moche y Santa. En Arqueología Mochica: Nuevos enfoques, L. J. Castillo, H. Bernier, G. Lockard \& J. Rucabado, Eds., pp. 203-217. Lima: Instituto Francés de Estudios Andinos y Fondo Editorial de la Pontificia Universidad Católica del Perú.

Gumerman, G. \& J. Briceño, 2003. Santa Rosa-Quirihuac y Ciudad de Dios: Dos asentamientos rurales en la parte media del valle de Moche. En Moche: Hacia el final del milenio, Tomo I, S. Uceda \& E. Mujica, Eds., pp. 217-243. Lima: Fondo Editorial de la Pontificia Universidad Católica del Perú.

Hocquenghem, A. M., 1987. Iconografía Mochica. Lima: Fondo Editorial de la Pontificia Universidad Católica del Perú.

Jackson, M., 2008. Moche Art and Visual Culture in Ancient Peru. Albuquerque: University of New Mexico Press.

LARCO, R., 1948. Cronología arqueológica del norte del Perú. Buenos Aires: Sociedad Geográfica Americana,

Lechtman, H., 1996. Cloth and Metal: The Culture of Technology. En Andean Art at Dumbarton Oaks, Vol. I, E. H. Boone, Ed. Washington, D.C.: Dumbarton Oaks Research Library and Collection.

Lechtman, H.; A. Erlij \& E. Barry, 1982. New Perspectives on Moche Metallurgy: Techniques of Gilded Copper at Loma Negra, Northern Peru. American Antiquity 47: 3-30.

Makowski, K., 2009. Virú-Moche Relations: Technological Identity, Stylistic Preferences, and the Ethnic Identity of Ceramic Manufacturers and Users. En Gallinazo. An Early Cultural Tradition on the Peruvian North Coast, J. F. Millaire \& M. Morlion, Eds., pp. 33-59. Los Angeles, CA: Cotsen Institute of Archaeology Press, University of California, Los Angeles.

PARsons, L., 1962. An Examination of Four Moche Jars from the Same Mold. American Antiquity 27 (4): 515-519.

Pernot, M., 2010. Técnicas del metal, artesanos y talleres en las sociedades antiguas. De la Edad del Bronce final al período romano en la Europa occidental. Bulletin de l'Institut Français d'Études Andines 39 (2): 331-350, Lima.

Pillsbury, J. (ED.), 2001. Moche Art and Archaeology in Ancient Peru. Washington, D. C.: National Gallery of Art, Center for Advanced Studies in the Visual Arts, Symposium Papers XL.

Pimentel, V. \& M. Paredes, 2003. Evidencias Moche V en tambos y caminos entre los valles de Santa y Chao, Perú. En Moche: Hacia el final del milenio, Tomo I, S. Uceda \& E. Mujica, Eds., pp. 269-303. Lima: Fondo Editorial de la Pontificia Universidad Católica del Perú.

Purin, S., 1983. Utilisation des Rayons-X pour l'observation des traces de fabrication sur cinq vases Mochicas. Bulletin des Musées Royaux d'Art et d'Histoire 54 (2): 5-20, Bruselas.

1985. Construction de trois vases noirs Mochicas. Bulletin des Musées Royaux d'Art et d'Histoire 56 (1): 95-104, Bruselas.

Quilter, J., 2010. Moche: Archaeology, Ethnicity, Identity. Bulletin de l'Institut Français d'Études Andines 39 (2): 225-241, Lima.

Quilter, J. \& L. J. Castillo (Eds.), 2010. New Perspectives on Moche Sociopolitical Organization. Washington, D. C.: Dumbarton Oaks Research Library and Collection.

Rohfritsch, A., 2010. Contribución arqueométrica al estudio de las técnicas y de la organización de la producción de cerámica ritual en la sociedad Mochica 8150-850 DC, costa norte del Perú. Bulletin de l'Institut Français d'Études Andines 39 (2): 389-412, Lima.

Russell, G.; B. LeOnARD \& J. BriceÑo, 1998. The Cerro Mayal Workshop: Addressing Issues of Craft Specialization in Moche Society. En Andean Ceramics: Technology, Organization, and Approaches, I. Shimada, Ed., pp. 63-89. Philadelphia, PA: University of Pennsylvania Museum of Archaeology and Anthropology.

Tello, R. \& T. Delabarde, 2008. Tumbas del Conjunto Arquitectónico 35 de Huacas del Sol y de la Luna. En Investigaciones en la 
Huaca de la Luna 2001, S. Uceda, E. Mujica \& R. Morales, Eds. pp. 129-173. Trujillo: Universidad Nacional de Trujillo.

Trever, L.; J. Gamboa, R. Toribio \& F. Surette, 2013. Moche Feathered Shield from the Painted Temples of Pañamarca. Ñawpa Pacha 33 (1): 1-17. Berkeley: Institute of Andean Studies.

Tufinio, M., 2006. Excavaciones en Plaza 2B de Huaca de la Luna En Investigaciones en la Huaca de la Luna 2001, S. Uceda, E. Mujica \& R. Morales, Eds., pp. 33-45. Trujillo: Universidad Nacional de Trujillo.

UCEDA, S. \& J. Armas, 1998. An Urban Pottery Workshop at the Site of Moche, North Coast of Peru. En Andean Ceramics: Technology, Organization, and Approaches, I. Shimada, Ed., pp. 91-110. Philadelphia, PA: University of Pennsylvania Museum of Archaeology and Anthropology.

UCEDA, S.; C. Chapdelaine \& J. Verano, 2008. Fechas radiocarbónicas para el Complejo Arqueológico Huacas del Sol y de la Luna.
En Investigaciones en la Huaca de la Luna 2001, S. Uceda, E. Mujica \& R. Morales, Eds. pp. 213-223. Trujillo: Universidad Nacional de Trujillo.

UCEDA, S. \& E. MujICA (EDs.), 1994. Moche: Propuestas y perspectivas. Actas del Primer Coloquio sobre la Cultura Moche. Travaux de l'Institute Français d'Études Andines 79. Lima: Universidad Nacional de Trujillo, Instituto Francés de Estudios Andinos y FOMCIENCIAS

- 2003. Moche: Hacia el final del milenio. Actas del Segundo Coloquio sobre la Cultura Moche. Lima: Fondo Editorial de la Pontificia Universidad Católica del Perú.

Wilson, D., 1988. Prehispanic Settlement Patterns in the Lower Santa Valley, Peru: A Regional Perspective on the Origins and Development of Complex North Coast Society Smithsonian Series in Archaeological Inquiry. Washington, D. C.: Smithsonian Institution Press. 
